\title{
Coping e engajamento no trabalho de equipe de enfermagem hospitalar
}

\section{Coping and work engagement in hospital nursing team Coping y compromiso del trabajo en equipo de enfermería hospitalaria}

\author{
Adaiane Amélia Baccin (iD \\ Universidade Federal de Santa Maria - Santa Maria (RS) - Brasil
}

Anniara Lúcia Dornelles de Lima (iD

Universidade Federal de Santa Maria - Santa Maria (RS) - Brasil

Juliana Kuster de Lima Maliska iD

Universidade Federal de Santa Maria - Santa Maria (RS) - Brasil

Leila Mara Piasentin Claro iD

Universidade Federal de Santa Maria - Santa Maria (RS) - Brasil

Vanessa Cirolini Lucchese iD

Universidade Federal de Santa Maria - Santa Maria (RS) - Brasil

Silvio José Lemos Vasconcellos iD

Universidade Federal de Santa Maria - Santa Maria (RS) - Brasil

\section{RESUMO}

Objetivo: Avaliar a relação entre coping e engajamento em profissionais de enfermagem de um hospital público. Métodos: Trata-se de um estudo quantitativo, descritivo, correlacional e transversal, realizado entre dezembro de 2017 a janeiro de 2018, com 250 trabalhadores da equipe de enfermagem em um hospital público localizado no interior do Rio Grande do Sul. Participaram técnicos em enfermagem e enfermeiros. Empregaram-se três instrumentos autoaplicáveis: a Escala de Coping Ocupacional, a Utrecht Work Engagement Scale e um questionário abrangendo variáveis sociodemográficas, desenvolvido pelos pesquisadores. Utilizou-se estatística descritiva e, para análise, correlação de Pearson, considerando significativos os valores de p<0,01. Resultados: Amostra composta por 139 (55,6\%) técnicos em enfermagem e $111(44,4 \%)$ enfermeiros, sendo 84\% $(n=$ $210)$ do sexo feminino. Apresentaram-se correlações positivas, fracas e significativas, principalmente entre coping planejamento e as dimensões em engajamento: vigor $(0,28)$, dedicação $(0,27)$, concentração $(0,27)$ e total $(0,30)$; e entre coping total e as dimensões em engajamento: vigor $(0,22)$, dedicação $(0,21)$ e total $(0,21)$. Conclusão: As correlações significativas encontradas entre coping e engajamento em profissionais da enfermagem demonstram a relevância da avaliação desses conceitos. Portanto, determinados fatores de coping ocupacional e engajamento podem auxiliar nas atividades laborais na equipe de enfermagem.

Descritores: Equipe de Enfermagem; Hospitais; Psicologia.

\begin{abstract}
Objective: Assess the relationship between coping and engagement in nursing professionals at a public hospital. Methods: This is a quantitative, descriptive, correlational, and cross-sectional study, carried out between December 2017 and January 2018 , with 250 nursing staff workers in a public hospital located in the interior of Rio Grande do Sul. Nursing technicians and nurses participated. Three self-administered instruments were used: The Occupational Coping Scale, the Utrecht Work Engagement Scale, and a questionnaire covering sociodemographic variables developed by the researchers. Descriptive statistics were used, and Pearson's correlation was used for analysis, considering $p$ values $<0.01$ as significant. Results: Sample composed of 139 (55.6\%) nursing technicians and $111(44.4 \%)$ nurses, 84\% ( $n=210)$ female. Positive, weak, and significant correlations were presented, mainly between coping planning and the dimensions in engagement: vigor (0.28), dedication (0.27), concentration (0.27) and total (0.30); and between total coping and engagement dimensions: vigor (0.22), dedication (0.21) and total (0.21). Conclusion: The significant correlations found between coping and engagement in nursing professionals demonstrate the relevance of evaluating these concepts. Therefore, certain occupational coping and engagement factors can help in the work activities of the nursing team.
\end{abstract}

Descriptors: Nursing Team; Hospitals; Psychology. 


\section{RESUMEN}

Objetivo: Evaluar la relación entre coping y compromiso de profesionales de enfermería de un hospital público. Métodos: Se trata de un estudio cuantitativo, descriptivo, correlacional y transversal realizado entre diciembre de 2017 y enero de 2018 con 250 trabajadores del equipo de enfermería de un hospital público localizado en el campo de Río Grande de Sur. Participaron técnicos de enfermería y enfermeros. Se ha aplicado tres instrumentos auto aplicables: la Escala de Coping Ocupacional, la Utrecht Work Engagement Scale y un cuestionario con variables sociodemográficas desarrollado por los investigadores. Se utilizó la estadística descriptiva y, para el análisis, la correlación de Pearson, considerando significativos los valores de $p<0,01$. Resultados: Muestra de 139 (55,6\%) técnicos de enfermería y 111 (44,4\%) enfermeros, de ellos el 84\% $(n=210)$ era del sexo femenino. Se ha encontrado correlaciones positivas, débiles y significativas, principalmente entre coping planeamiento y las dimensiones del compromiso: vigor $(0,28)$, dedicación $(0,27)$, concentración $(0,27)$ y total $(0,30)$; y entre coping total y las dimensiones del compromiso: vigor $(0,22)$, dedicación $(0,21)$ y total $(0,21)$. Conclusión: Las correlaciones significativas encontradas entre coping y compromiso de profesionales de enfermería han demostrado la relevancia de la evaluación de esos conceptos. Por lo tanto, determinados factores de coping ocupacional y compromiso pueden ayudar en las actividades laborales del equipo de enfermería.

Descriptores: Grupo de Enfermería; Hospitales; Psicología.

\section{INTRODUÇÃO}

O trabalho, no sentido laboral, pode assumir uma atribuição muito importante no cotidiano das pessoas, podendo ser gerador de realizações, como também ocasionar adoecimentos. Desse modo, estudar as formas de enfrentar o estresse e evitar adoecimento na área profissional são alvos de investigação das ciências humanas, sociais e da saúde. Pesquisas relacionando as temáticas estresse e coping, entre profissionais da saúde, revelam resultados preocupantes sobre os altos índices de estresse nesses trabalhadores e no contexto laboral ${ }^{(1-4)}$.

Ao conceituar coping, entende-se que há uma diferenciação principal entre duas abordagens, sendo elas: a disposicional e a transacional( ${ }^{(5)}$. No presente artigo, o foco recai sobre a abordagem disposicional, que analisa $o$ coping como um estilo e é considerado como constituição de personalidade do indivíduo que sobressai à influência do tempo ou outros fatores, evidenciando a estabilidade dos comportamentos relacionados ao enfrentamento de situações estressantes ${ }^{(5,6)}$.

O engajamento no trabalho é determinado como um estado mental positivo, não ocorrendo somente em situações breves, que se estabelece por apresentar elevado grau de energia e realização, já que é uma experiência positiva e gratificante relacionada ao desempenho das tarefas no trabalho ${ }^{(7)}$. Desse modo, o engajamento pode ser representado por três bases principais: dedicação, concentração e vigor ${ }^{(7)}$.

Nesse sentido, com foco no coping e no engajamento no trabalho, pode-se atuar com vistas a promover a saúde em ambientes de trabalho de profissionais da saúde. Significativos ganhos podem tornar-se consequências, por meio do equilíbrio entre as exigências do ambiente de trabalho e as habilidades do profissional, viabilizando assim aspectos positivos relativos à atuação do trabalhador ${ }^{(8)}$.

No que diz respeito ao trabalho da enfermagem, pode-se inferir que o mesmo abrange um contexto dinâmico, envolvendo diferentes tarefas em áreas como promoção de saúde, prevenção, tratamentos e reabilitações ${ }^{(9,10)}$. Dessa forma, na literatura especializada, estudos ponderam sobre os diversos adoecimentos que essa categoria pode experienciar no ambiente detrabalho( ${ }^{(2,11,12)}$.

A temática do estresse e do coping, por exemplo, apresentou-se como alvo de pesquisa internacional com estudantes de graduação em obstetrícia e enfermagem, analisada por meio de uma revisão integrativa contemplando um total de vinte e cinco artigos ${ }^{(13)}$. Destaca-se, na pesquisa citada, a escassez de direcionamento e instrução, por parte dos educadores, quanto ao modo de lidar com eventos estressantes vivenciados pelos alunos durante 0 período de formação ${ }^{(13)}$.

Por intermédio de uma revisão sistemática da literatura, indicou-se que, em instituições de saúde, há grande incidência da Síndrome de Burnout, sendo a equipe de enfermagem a categoria mais afetada, já que, geralmente, demonstra-se ser o grupo que ocupa majoritariamente esses espaços ${ }^{(3)}$. Tendo em vista que esses profissionais são uma população com grande possibilidade de adoecimentos, demonstra-se a necessidade de entender aspectos no contexto laboral que possam auxiliar nestas demandas.

Nesse cenário, a análise da relação entre fatores de coping e engajamento, no ambiente de trabalho, poderá contribuir para estratégias de intervenção e de planejamento de ações para a promoção da saúde de trabalhadores 
de enfermagem ${ }^{(1,3,14)}$. Além disso, o estudo justifica-se pela importância em contribuir com resultados que ampliam essa discussão, e tem como foco uma maior valorização e reconhecimento do trabalho da equipe de enfermagem, tendo em vista a importância do seu papel na organização e no cuidado dos pacientes ${ }^{(2)}$.

Desse modo, a presente pesquisa tem como foco avaliar a relação entre coping ocupacional e engajamento no trabalho em profissionais de enfermagem de um hospital público.

\section{MÉTODOS}

Trata-se de um estudo quantitativo, descritivo, correlacional e transversal desenvolvido de 12 de dezembro de 2017 a 12 de janeiro de 2018, com profissionais da enfermagem atuantes de um hospital público, localizado no interior do Rio Grande do Sul. A amostragem deu-se por conveniência. Para participar da pesquisa selecionaram-se 250 profissionais, sendo os mesmos enfermeiros e técnicos de enfermagem. Definiu-se o total de participantes, considerando uma população total de 952 trabalhadores e um erro amostral de 5\%.

Como critérios de inclusão, o profissional deveria ser membro da equipe de enfermagem há pelo menos um ano, pelo fato de já ter experienciado alguma situação de estresse. Para os critérios de exclusão demarcou-se estar afastado das atividades de trabalho no período, seja por motivo de atestado médico ou em férias, e estar atuando no hospital há menos de um ano.

A coleta de dados ocorreu a partir dos instrumentos impressos e aplicados pessoalmente no hospital pelos pesquisadores do presente estudo, durante o turno de trabalho dos profissionais. Os instrumentos eram autoaplicáveis, favorecendo assim a participação na pesquisa sem prejudicar a rotina laboral. De maneira individual ou coletiva, os pesquisadores abordavam os trabalhadores, comunicavam as informações relativas aos objetivos da pesquisa, critérios de inclusão e instruções de preenchimento e, em seguida, os convidavam para participar. Após o aceite, o Termo de Consentimento Livre e Esclarecido (TCLE) era lido e assinado por cada participante, ficando um de posse do participante e outro com o pesquisador.

A maioria dos participantes respondeu a pesquisa no momento da abordagem, sendo o tempo de aplicação de cerca de 20 minutos. Entregaram-se os instrumentos, para posterior recolhimento, apenas aos participantes que integravam equipes em que não era possível a permanência de pessoas externas no setor de trabalho, como no Centro Cirúrgico, por exemplo.

Empregaram-se três instrumentos: um questionário contendo perguntas sociodemográficas, desenvolvido pelos autores; a Escala validada( ${ }^{(5)}$ de Coping Ocupacional (ESCO) e autorizada para uso nesta pesquisa, e a Utrecht Work Engagement Scale (UWES) já publicada, validada e adaptada para o Brasil(15). A Escala de Coping Ocupacional (ESCO) é constituída por uma escala do tipo Likert (de 0 a 3 pontos), com quatro opções possíveis (nunca, raramente, às vezes e sempre) para a análise de 24 itens ${ }^{(5)}$. Esses itens são divididos em quatro dimensões: Planejamento, Emoções Negativas, Interação Social e Evitação. Além disso, adequou-se o alfa de Cronbach para o fator geral $(\alpha=0,70)^{(5)}$.

Ainda na Escala de Coping Ocupacional (ESCO), o Fator Planejamento é constituído por 10 itens e composto por elementos que seguem aspectos de maior objetividade em direção à resolução dos problemas ${ }^{(5)}$. O Fator Emoções Negativas é composto por 7 itens que se referem às ações negativas realizadas, tanto afetivas quanto comportamentais, diante das diversidades no trabalho ${ }^{(5)}$.

O Fator Interação Social engloba 4 itens relacionados à comunicação com outras pessoas, como forma de apoio e auxílio em percepções de possíveis formas de resolução de problemas no trabalho ${ }^{(5)}$. O Fator Evitação contém 3 itens, apresentando elementos correspondentes a reagir de forma evitativa no que se refere às dificuldades apresentadas no trabalho(5).

A Utrecht Work Engagement Scale (UWES) é constituída por 17 itens $(n=17)$ avaliados por uma escala tipo Likert (de 0 a 6 pontos), de sete alternativas (0. nunca, 1. quase nunca, 2. raramente, 3. algumas vezes, 4. frequentemente, 5 . com muita frequência e 6 . sempre), com base na perspectiva teórica chamada de recursos e demandas de trabalho $(\mathrm{RDT})^{(15)}$. As propriedades psicométricas da escala indicam 3 dimensões para o instrumento: vigor, dedicação e concentração ${ }^{(7)}$. O coeficiente alfa de Cronbach para o fator geral é de $\alpha=0,95$, demonstrando a adequação da escala ${ }^{(15)}$.

As dimensões vigor, dedicação e concentração, utilizadas na estrutura fatorial da escala, apresentam as seguintes características: o vigor é medido em relação à energia e foco colocados no trabalho, as quais persistem mesmo se algo não dá certo; já a dedicação descreve o propósito atribuído ao trabalho no qual gera realização profissional; e a concentração representa o envolvimento intenso ao fazer as atividades, vinculando-se totalmente com o prazer 
ao trabalho que está realizando. Assim as dimensões juntas demonstram o engajamento no trabalho, como estado mental positivo de bem-estar e realizações ${ }^{(7,15)}$.

Utilizou-se o programa Statistical Package for Social Sciences (SPSS), versão 20.0, para análise dos dados. E empregou-se a estatística descritiva e a correlação de Pearson, considerando significativos os valores de $p<0,01$.

Para a realização da pesquisa, seguiram-se os preceitos da Resolução n. ${ }^{\circ} 466 / 12$ do Conselho Nacional de Saúde do Ministério da Saúde. Além disso, o projeto obteve aprovação do Comitê de Ética em Pesquisa da Universidade Federal de Santa Maria, sob Parecer $n^{\circ} 3.088 .518$.

\section{RESULTADOS}

A seguir, são apresentados os dados por intermédio de tabelas para uma melhor visualização. No que diz respeito à amostra resultante, houve maior participação de técnicos de enfermagem $(55,6 \% ; n=139)$ do que enfermeiros $(44,4 \% ; n=111)$ e predominância de participantes do sexo feminino $(84 \% ; n=210)$. Apresentaram-se idades entre 23 e 64 anos, com média de 40 anos $( \pm 9,5)$. Assim, na presente pesquisa, a caracterização geral do perfil da amostra ficou definida conforme apresenta a Tabela I.

Tabela I - Características sociodemográficas dos profissionais da equipe de enfermagem. Rio Grande do Sul, Brasil, 2018.

\begin{tabular}{llcc}
\hline Variável & \multicolumn{1}{c}{ Alternativas } & Frequência & Percentual \\
\hline Cargo & Técnico(a) em Enfermagem & 139 & $55,6 \%$ \\
& Enfermeiro(a) & 111 & $44,4 \%$ \\
Sexo & Feminino & 210 & $84 \%$ \\
& Masculino & 40 & $16 \%$ \\
Faixa Etária & Até 25 anos & 4 & $1,6 \%$ \\
& De 25 a 35 & 79 & $31,6 \%$ \\
& De 35 a 45 & 101 & $40,4 \%$ \\
& De 45 a 60 anos & 59 & $23,6 \%$ \\
& Acima de 60 anos & 7 & $2,8 \%$ \\
\hline
\end{tabular}

Na Tabela Il é apresentado o resultado das correlações entre as variáveis dos instrumentos ESCO e UWES. As correlações são positivas e fracas, porém significativas entre coping planejamento e as dimensões de engajamento. Assim temos que: vigor $(0,28)$, dedicação $(0,27)$, concentração $(0,27)$ e total $(0,30)$. Já entre coping total e as dimensões de engajamento tem-se: vigor $(0,22)$, dedicação $(0,21)$ e total $(0,21)$. Quanto ao coping emoções negativas com engajamento, a correlação apresentou-se como positiva, fraca e significativa apenas com vigor $(0,21)$. Os fatores coping interação social e coping evitação não demonstraram correlações significativas com nenhuma dimensão de engajamento.

Tabela II - Correlações entre os fatores da Escala de Coping Ocupacional (ESCO) e as dimensões da Utrecht Work Engagement (UWES). Rio Grande do Sul, Brasil, 2018.

\begin{tabular}{lcccc}
\hline & $\begin{array}{c}\text { Engajamento } \\
\text { Vigor } \\
\mathbf{r}\end{array}$ & $\begin{array}{c}\text { Engajamento } \\
\text { Dedicação } \\
\mathbf{r}\end{array}$ & $\begin{array}{c}\text { Engajamento } \\
\text { Concentração } \\
\mathbf{r}\end{array}$ & $\begin{array}{c}\text { Engajamento } \\
\text { Total } \\
\mathbf{r}\end{array}$ \\
\hline Coping Planejamento & $+0,28^{* *}$ & $0,27^{* *}$ & $0,27^{* *}$ & $0,30^{* *}$ \\
Coping Interação Social & $-0,07$ & $-0,00$ & $-0,00$ & $-0,03$ \\
Coping Emoções negativas & $+0,21^{* *}$ & $0,14^{*}$ & 0,04 & $0,14^{*}$ \\
Coping Evitação & $-0,01$ & $-0,00$ & $-0,06$ & $-0,03$ \\
Coping Total & $+0,22^{* *}$ & $0,21^{* *}$ & $0,14^{*}$ & $0,21^{* *}$ \\
\hline
\end{tabular}

${ }^{*} p<0,05 ;{ }^{*} p<0,01 ;$;: Teste de correlação de Pearson 


\section{DISCUSSÃO}

Demonstra-se que houve um grande percentual do sexo feminino na amostra (84\%) da presente pesquisa, o que é uma característica frequente no contexto da enfermagem ${ }^{(2,11)}$. Também ocorreu maior participação de técnicos de enfermagem do que enfermeiros. Isso pode ser explicado considerando o fato de que técnicos se caracterizam por ter um grande contingente de profissionais que atuam em hospitais ${ }^{(16)}$.

Em relação às correlações entre coping e engajamento, a ocorrência de uma correlação positiva e estatisticamente significativa, porém fraca, deu-se entre coping planejamento e as diferentes dimensões de engajamento. Esse resultado pode, até certo ponto, estar relacionado com a busca por estratégias mais ativas de coping em relação ao problema por profissionais engajados. A correlação, por exemplo, entre o engajamento total com coping planejamento $(0,30)$, que na $\mathrm{ESCO}^{(5)}$ engloba características do tipo: pensa que desafios são maneiras de superar; identifica que frente a grandes dificuldades pode ocorrer maior planejamento de pessoas engajadas no ambiente laborativo.

Esse achado vai ao encontro dos resultados de estudo realizado com 310 participantes, que investigou quais estratégias de coping eram mais usadas por auxiliares e técnicos de enfermagem de um hospital geral ${ }^{(17)}$. $O$ resultado do estudo demonstrou que 58,4\% usavam estratégias direcionadas para o problema, tornando-se o tipo de estratégia mais empregada pela amostra ${ }^{(17)}$. Como forma de amparo contra o estresse, identifica-se a relevância na elaboração de capacitações de estratégias de coping referentes à resolução de problemas direcionadas aos trabalhadores de enfermagem ${ }^{(18,19)}$.

Quanto ao construto coping emoções negativas e a respectiva correlação com engajamento, houve índice positivo, fraco e estatisticamente significativo apenas com a dimensão vigor. Conforme mencionado anteriormente, as emoções negativas representam a forma de avaliar de modo emotivo a situação, qualificando-a negativamente e podendo gerar comportamentos desfavoráveis como irritar-se, ficar agressivo, impulsivo ou culpar-se ${ }^{(5,6)}$.

Entretanto, emoções negativas não se apresentam como formas eficientes de aliviar o estresse ${ }^{(20)}$. Em pesquisa realizada com 1.806 enfermeiros poloneses, verificou-se, por meio de coeficientes de correlação, que atitudes como, por exemplo, culpar-se e negação tiveram vinculação positiva, fraca e significativa com a manifestação de síndrome de burnout no ambiente de trabalho(20).

No que diz respeito à relação coping interação social e coping evitação, salienta-se que não se observou correlações significativas com engajamento em nenhum dos fatores no presente estudo. Essa ausência de correlação pode ser entendida pela questão do engajamento poder ser sujeito a alterações vinculadas a várias condições ${ }^{(21)}$. O engajamento demonstra-se ser formado por vários aspectos entrelaçados por conhecimentos adquiridos tanto no âmbito pessoal quanto nas atividades laborais e por condições disposicionais, podendo, dessa forma, variar ${ }^{(21)}$.

Quanto às correlações entre coping total com engajamento, salienta-se que as mesmas mostraram-se positivas e significativas para vigor, dedicação e para engajamento total no presente estudo. Tais correlações, embora fracas, podem indicar que, entre as categorias, há uma relação sugestiva entre coping total e engajamento, apresentando a possibilidade de as duas tendências serem necessárias para uma melhor interação no ambiente de trabalho. Vale destacar que, em estudo semelhante, demonstrou-se a relação de menores índices de estresse com a utilização de estratégias de coping adaptativas pelos enfermeiros ${ }^{(20)}$. Esses dados vão ao encontro de trabalhos que obtiveram dados similares, nos quais demonstraram relações significativas entre fatores psicossociais positivos no ambiente do trabalho e engajamento em enfermeiros, proporcionando um ambiente mais assertivo aos seus funcionários ${ }^{(21-23)}$. Dessa maneira, a relação coping e engajamento parece, de forma conjunta, fundamentar uma melhor condição em atividade laboral para a equipe de enfermagem.

Em vista disso, evidencia-se, no presente estudo, a correlação positiva entre o coping ocupacional, referindo-se à coping planejamento e à coping total, com as dimensões de engajamento. Por meio disso, a ampliação do conhecimento sobre o tema pode fomentar intervenções focadas em táticas de coping mais eficazes e aprimoração de engajamento para profissionais de enfermagem ${ }^{(18,19,24,25)}$.

Logo, é importante destacar que para que a equipe de enfermagem possa ter a possibilidade de exercer o serviço de forma hábil à população, o bem-estar no trabalho, incluindo fatores de coping e engajamento, devem ser levados em consideração ${ }^{(18,25,26)}$. Em vista disso, estudos como o realizado na presente pesquisa são necessários para que assim se possa estabelecer as possíveis dificuldades que os trabalhadores estão enfrentando e as potencialidades no contexto laboral ${ }^{(14)}$.

Salienta-se que o presente estudo apresentou determinadas limitações. Devido ao fato da pesquisa ser aplicada apenas em um hospital, os dados obtidos podem não se estender a outras instituições e cenários. Além disso, em virtude do estudo ser de natureza transversal e correlacional, não é possível inferir causalidade nos resultados. 


\section{CONCLUSÃO}

Averiguaram-se correlações positivas, significativas, porém fracas, principalmente entre coping planejamento e coping total com dimensões de engajamento. Dessa forma, determinados fatores de coping ocupacional e engajamento podem auxiliar nas atividades laborais na equipe de enfermagem.

\section{CONFLITOS DE INTERESSE}

Os autores declaram não haver conflitos de interesses.

\section{CONTRIBUIÇÕES}

Adaiane Amélia Baccin contribuiu com a elaboração e delineamento do estudo; aquisição, análise e interpretação de dados; e a redação e/ou revisão do manuscrito. Anniara Lúcia Dornelles de Lima,Vanessa Cirolini Lucchese e Silvio José Lemos Vasconcellos contribuíram com a aquisição, análise e interpretação de dados; e a redação e/ou revisão do manuscrito. Juliana Kuster de Lima Maliska e Leila Mara Piasentin Claro contribuíram com a aquisição, análise e interpretação de dados. Todos os autores aprovaram a versão final a ser publicada e são responsáveis por seu conteúdo.

Manuscrito baseado na dissertação de mestrado intitulada: "Coping e Engajamento no Trabalho da Enfermagem Hospitalar", ano 2018, 76 páginas.

\section{REFERÊNCIAS}

1. Al Barmawi MA, Subih M, Salameh O, Sayyah Yousef Sayyah N, Shoqirat N, Abdel-Azeez Eid Abu Jebbeh R. Coping strategies as moderating factors to compassion fatigue among critical care nurses. Brain Behav [Internet]. 2019 [acesso em 2020 Jul 14];9(4):e01264. Disponível em: https://www.ncbi.nlm.nih.gov/pmc/ articles/PMC6456805/pdf/BRB3-9-e01264.pdf

2. Hämmig $O$. Explaining burnout and the intention to leave the profession among health professionals - a crosssectional study in a hospital setting in Switzerland. BMC Health Serv Res [Internet]. 2018 [acesso em 2020 Ago 18];18(1):785. Disponível em: https://www.ncbi.nlm.nih.gov/pmc/articles/PMC6194554/pdf/12913_2018_ Article_3556.pdf

3. Friganović $A$, Selič $P$, llić $B$, Sedić $B$. Stress and burnout syndrome and their associations with coping and job satisfaction in critical care nurses: a literature review. Psychiatr Danub. 2019;31(Suppl 1):21-31.

4. Borges EMN, Fonseca CINS, Baptista PCP, Queirós CML, Baldonedo-Mosteiro M, Mosteiro-Diaz MP. Fadiga por compaixão em enfermeiros de urgência e emergência hospitalar de adultos. Rev Lat Am Enfermagem [Internet]. 2019 [acesso em 2020 Jul 11];27:e3175. Disponível em: http://www.scielo.br/scielo.php?script=sci_ arttext\&pid=S0104-11692019000100360\&lng=en

5. Alves GAS. Construção de uma Escala de Coping Ocupacional (ESCO): Estudos psicométricos preliminares [dissertação]. Itatiba: Universidade São Francisco; 2010.

6. Lazarus RS, Folkman S. Stress, appraisal, and coping. New York: Springer; 1984.

7. Schaufeli WB, Salanova M, González-Romá V, Bakker AB. The measurement of engagement and burnout: a two sample confirmatory factor analytic approach. J Happiness Stud. 2002;3:71-92.

8. Farsen TC, Boehs STM, Ribeiro ADS, Biavati VP, Silva N. Qualidade de vida, Bem-estar e Felicidade no Trabalho: sinônimos ou conceitos que se diferenciam? Interação Psicol [Internet]. 2018 [acesso em 2020 Jun 17];22(1):31-41. Disponível em: https://revistas.ufpr.br/psicologia/article/view/48288/35057

9. Toso GL, Golle L, Magnago TSBS, Herr GEG, Loro MM, Aozane F, et al. Cultura de segurança do paciente em instituições hospitalares na perspectiva da enfermagem. Rev Gaúch Enferm [Internet]. 2016 [acesso em 2020 Jun 21];37(4):e58662. Disponível em: http://www.scielo.br/scielo.php?script=sci_arttext\&pid=S1983$14472016000400405 \&$ Ing $=$ en

10. Silva RM, Zeitoune RCG, Beck CLC, Martino MMF, Prestes FC. Efeitos do trabalho na saúde de enfermeiros 
que atuam em clínica cirúrgica de hospitais universitários. Rev Lat Am Enfermagem [Internet]. 2016 [acesso em 2020 Ago 14];24:e2743. Disponível em: https://www.scielo.br/pdf/rlae/v24/pt_0104-1169-rlae-24-02743. pdf

11. Javadi-Pashaki N, Darvishpour A. Survey of stress and coping strategies to predict the general health of nursing staff. J Educ Health Promot [Internet]. 2019 [acesso em 2020 Ago 18];8:74. Disponível em: https:// www.ncbi.nlm.nih.gov/pmc/articles/PMC6512229/

12. Dyrbye LN, Shanafelt TD, Johnson PO, Johnson LA, Satele D, West CP. A cross-sectional study exploring the relationship between burnout, absenteeism, and job performance among American nurses. BMC Nurs [Internet]. 2019 [acesso em 2021 Ago 02];18:57. Disponível em: https://bmcnurs.biomedcentral.com/track/ pdf/10.1186/s12912-019-0382-7.pdf

13. McCarthy B, Trace A, O'Donovan M, Brady-Nevin C, Murphy M, O'Shea M, et al. Nursing and midwifery students' stress and coping during their undergraduate education programmes: an integrative review. Nurse Educ Today. 2018;61:197-209.

14. Lourenção LG. Work engagement among participants of residency and professional development programs in nursing. Rev Bras Enferm [Internet]. 2018 [acesso em 2020 Ago 15];71(Suppl 4):1487-92. Disponível em: http://www.scielo.br/scielo.php?script=sci_arttext\&pid=S0034-71672018001001487\&lng=en

15. Vazquez ACS, Magnan ES, Pacico JC, Hutz CS, Schaufeli WB. Adaptation and Validation of the Brazilian Version of the Utrecht Work Engagement Scale. Psico-USF [Internet]. 2015 [acesso em 2020 Jun 18];20(2):207-17. Disponível em: https://www.scielo.br/j/pusf/a/94kdp9kC8fHkphG8yP6zgpH/?lang=en

16. Marques JPD, Beck CLC, Silva RM, Prestes FC, Coelho APF, Vedootto DO. Satisfação no trabalho hospitalar de técnicos de Enfermagem com formação superior. REME Rev Min Enferm [Internet]. 2017 [acesso em 2021 Ago 05];21:e-1061. Disponível em: http://reme.org.br/artigo/detalhes/1199

17. Pereira SS, Teixeira CAB, Reisdorfer E, Vieira MV, Gherardi-Donato ECS, Cardoso L. The relationship between occupational stressors and coping strategies in nursing technicians. Texto \& Contexto Enferm [Internet]. 2016 [acesso em 2020 Jul 11];25(4):e2920014. Disponível em: http://www.scielo.br/scielo. php?script=sci_arttext\&pid=S0104-07072016000400331\&lng=en

18. Sampaio CL, Almeida PC, Souza AMA, Neri MFS, Silva LA, Caetano JA. Differences between quality of life and occupational coping of tenured and outsourced nurses. Rev Bras Enferm [Internet]. 2020 [acesso em 2021 Ago 7];73(Suppl 1):e20190462. Disponível em: https://www.scielo.br/j/reben/a/ tNS8HmymNPwmdrjnhfdmYpx/abstract/?lang=en\#

19. Schreuder JAH, Plat N, Magerøy N, Moen BE, van der Klink JJL, Groothoff JW et al. Self-rated coping styles and registered sickness absence among nurses working in hospital care: a prospective 1-year cohort study. Int J Nurs Stud. 2011;48(7):838-46.

20. Kupcewicz E, Jóźwik M. Positive Orientation and Strategies for Coping with Stress as Predictors of Professional Burnout among Polish Nurses. Int J Environ Res Public Health [Internet]. 2019 [acesso em 2020 Ago 15];16(21):4264. Disponível em: https://www.ncbi.nlm.nih.gov/pmc/articles/PMC6862310/

21. García-Sierra R, Fernández-Castro J, Martínez-Zaragoza F. Work engagement in nursing: an integrative review of the literature. J Nurs Manag. 2016;24(2):E101-E111.

22. Kunie K, Kawakami N, Shimazu A, Yonekura Y, Miyamoto Y. The relationship between work engagement and psychological distress of hospital nurses and the perceived communication behaviors of their nurse managers: a cross-sectional survey. Int J Nurs Stud. 2017;71:115-124.

23. Elliott R, Fry M. Psychological capital, well-being, and patient safety attitudes of nurses and midwives: a cross-sectional survey. Nurs Health Sci [Internet]. 2021 [acesso em 2021 Ago 05];23(1):237-44. Disponível em: https://onlinelibrary.wiley.com/doi/epdf/10.1111/nhs.12808

24. Moura LRNI, Charão-Brito L, Lopes LFD. Vigor, dedicação, absorção: uma análise da percepção de profissionais pós-graduandos sobre engajamento no trabalho. Rev Cesumar [Internet]. 2017 [acesso em 2020 Ago 10];22(2):229-45. Disponível em: https://periodicos.unicesumar.edu.br/index.php/revcesumar/ article/view/5706/3104 
25. Cao Y, Liu J, Liu K, Yang M, Liu Y. The mediating role of organizational commitment between calling and work engagement of nurses: a cross-sectional study. Int J Nurs Sci [Internet]. 2019 [acesso em 2021 Ago 4];6(3):309-14. Disponível em: https://www.ncbi.nlm.nih.gov/pmc/articles/PMC6722477/

26. Ugwu FO, Onyishi IE. The Moderating Role of Person-Environment Fit on the Relationship between Perceived Workload and Work Engagement among Hospital Nurses. Int J Afr Nurs Sci. 2020;13:100225.

\section{Endereço para correspondência:}

Adaiane Amélia Baccin

Universidade Federal de Santa Maria

Av. Roraima, 1000

Bairro: Camobi

CEP: 97105-900 - Santa Maria - RS - Brasil

E-mail: adaiane.baccin@gmail.com

Como citar: Baccin AA, Lima ALD, J Maliska JKL, Claro LMP, Lucchese VC, Vasconcellos SJL. Coping e engajamento no trabalho de equipe de enfermagem hospitalar. Rev Bras Promoç Saúde. 2021;34:11734. 